\title{
3-axis high Q MEMS accelerometer with simultaneous damping control
}

\author{
Ciotîrcă Lavinia $^{+*}$ Bernal Olivier $^{+}$, Tap Hélène ${ }^{+}$ \\ ${ }^{+}$CNRS, LAAS, 7 Avenue du Colonel Roche \\ Université de Toulouse - INPT \\ 31400 Toulouse, France \\ Lavinia.ciotirca@nxp.com
}

\author{
Enjalbert Jérôme*, Cassagnes Thierry ${ }^{*}$ \\ "NXP Semiconductors \\ 134 Avenue du General Eisenhower, BP 72329 \\ 31023 Toulouse Cedex 01, France \\ \{Jerome.enjalbert, Thierry.cassagnes\}@nxp.com
}

\begin{abstract}
The increasing demand of consumer market sensors involves a continuous development for the die size, cost and performances. In this context, the integration of both a 3-axis MEMS accelerometer and a gyroscope within the same low pressure cavity becomes attractive. Such a packaging results in MEMS with high quality factor $Q$, which reduces Brownian noise, thereby improving the achievable precision. However, contrary to gyroscopes, in such a configuration, the MEMS accelerometers need to be damped prior to measurement. Therefore, this paper presents a new damping control architecture for such high $Q$ capacitive accelerometers. Damping is ensured by inserting electrostatic force feedback (EFF) phases between measurement phases. By simultaneously damping 3 axis of acceleration, the transducer settling time is improved. Model simulations results prove damping efficiency and figure out a tradeoff between system sampling frequency and circuit complexity.
\end{abstract}

Keywords-Inertial sensors, MEMS accelerometers, electrostatic damping, multi-axis simultaneous control

\section{INTRODUCTION}

In the Internet of Things (IoT) era, sensing multiple degrees of freedom (DoF) with a unique integrated circuit (IC) becomes more and more attractive. MEMS capacitive inertial sensors (e.g. accelerometers, gyroscopes) provide high performance, low power, low die cost solutions for consumer market electronics and gain growing popularity. Therefore, a MEMS accelerometer and gyroscope fusion will be even more interesting. However, MEMS accelerometers are usually operating under atmospheric pressure while gyroscopes operate at high level vacuum. Consequently, the integration of both transducers within the same low pressure and high quality factor Q cavity necessitates a method to control ringing, by increasing the damping factor of the MEMS accelerometer.

Several closed loop control techniques, such as electrostatic damping, for capacitive MEMS accelerometers, were presented in [1]. Two different approaches can be considered: a $\Sigma \Delta$ loop (digital) and superimposing two electrostatic forces on the proof mass which results in a linear relationship (analogue). Both analogue and digital transducers implement electrostatic force control by applying a feedback voltage on the electrodes that are also used for read-out.
Although $\Sigma \Delta$ interfaces can provide high resolution digital output [2], [3], they have noise shaping issues and a very complicated implementation for a high order electromechanical closed loop.

Analogue transducers [4], [5] implement electrostatic damping for high Q MEMS capacitive accelerometers. The control voltage $V_{c t r l}$ is generated using a proportional derivative block and is applied on the sensors middle plate. The same electrodes are used during measurement and electrostatic force feedback phases and the circuits are designed for sensing one single acceleration direction.

In this paper, a new electrostatic damping architecture, which allows sensing and controlling more than one axis of acceleration, is presented. For a fully differential specific MEMS design that shares its middle plate between 3-axis: $x, y$ and $z$, and has only one pair of excitation electrodes for each axis, a new interface was designed in order to enable low power, low surface and high performance results. The read-out circuit is multiplexed between the 3 -axis and a simultaneous novel multi-rate damping technique is applied, to improve both the settling time and the system efficiency.

This paper is organized as follows: Section 2 describes the novel system architecture. The simultaneous damping control theory and the related chronograms are presented in detail in Section 3. Model simulation results are discussed in Section 4, followed by the conclusion in Section 5 .

\section{NOVEL SYSTEM DESIGN}

MEMS accelerometers can be modeled using a second order mass spring damper equation (1). In the presence of an external acceleration $a_{\text {ext }}$, the proof mass $m$ will deflect from its equilibrium position with a certain displacement $x$.

$$
m a_{\text {ext }}=m \ddot{x}+b \dot{x}+k x
$$

where $k$ is the spring constant and $b$ the damping coefficient.

When the MEMS cavity pressure starts decreasing, the quality factor $\mathrm{Q}$ will increase, causing oscillations that can result in measurement perturbations and even in part physical destruction if the oscillation amplitude is large enough. To avoid the above mentioned inconveniencies, one would like to develop a control system that damps the transducer, which implies increasing $b$. By adding a term proportional to the proof 
mass velocity $\dot{x}$ in (2), the damping coefficient will be increased by a factor $B$.

$$
m a_{e x t}=m \ddot{x}+(b+B) \dot{x}+k x
$$

The electrostatic damping principle consists in generating an electrical damping force (3) that will assist the mechanical damper.

$$
\Delta F=B \dot{x}
$$

The MEMS structure for one axis comprises three electrodes: one movable electrode, or the proof mass, and two fixed excitation electrodes (Fig.1). In the presence of external acceleration, the proof mass moves, which induces a capacitance variation between these electrodes. Moreover, when a voltage $\left(V_{\text {ex1 }}, V_{\text {exz }}\right.$ and $\left.V_{m}\right)$ is applied on the electrodes, an electrostatic force $\left(F_{1}, F_{2}\right)$ is generated between the proof mass and the excitation electrodes.

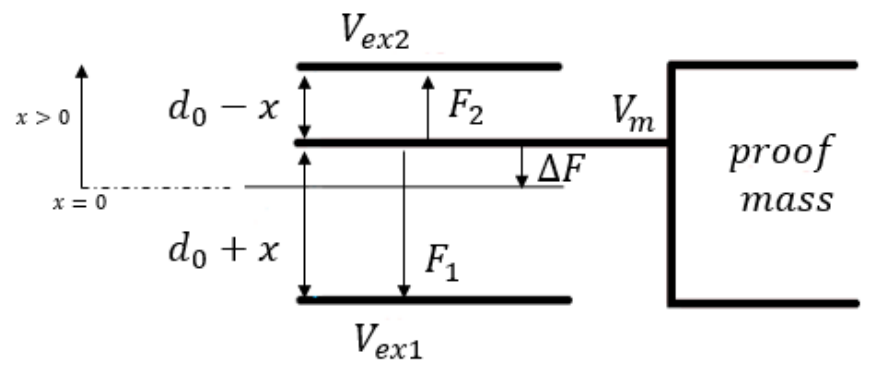

Fig.1 Parallel plate model of a capacitive sensor

The net electrostatic force $\Delta F$, detailed using equation (4) and applied to the proof mass, is an attractive force.

$$
\Delta F=F_{1}-F_{2}=\frac{1}{2} \varepsilon_{0} \varepsilon_{r} A\left(\frac{\left(V_{e x 1}-V_{m}\right)^{2}}{\left(d_{0}+x\right)^{2}}-\frac{\left(V_{e x 2}-V_{m}\right)^{2}}{\left(d_{0}-x\right)^{2}}\right)
$$

where $d_{0}$ is the gap at rest between the fixed electrodes and the proof mass and $x$ the proof mass displacement, $A$ is the total sense surface, $\varepsilon_{0}$ the vacuum permittivity and $\varepsilon_{r}$ the relative vacuum permittivity.

For a parallel plate capacitive sensor, when the voltage difference between the fixed plates and the middle one is 0 , no electrostatic force is applied on the proof mass. Supposing now that on the fixed plates, a differential bias $\pm V_{B}$ and an $\mathrm{AC}$ signal is superimposed on the common mode voltage $V_{m}$ as in (5), the net electrostatic force applied on the MEMS will depend on $V_{B}$ and $V_{c t r l}$.

$$
\begin{aligned}
& V_{\text {ex } 1}\left(\omega_{0} t\right)=V_{m}+V_{c t r l} \sin \left(\omega_{0} t\right)+V_{B} \\
& V_{\text {ex } 2}\left(\omega_{0} t\right)=V_{m}+V_{c t r l} \sin \left(\omega_{0} t\right)-V_{B}
\end{aligned}
$$

Moreover, if the control voltage $V_{c t r l}$ is the proof mass velocity estimation, then the electrostatic force will be proportional to the velocity and in (2) a new term is artificially added to assist the mechanical damping. The force generated using a control block, during a predefined damping time $t_{\text {damp }}$ within the sampling period $T_{s}$ is presented in (6). This force approximation is valid for very small proof mass displacements $x$ compared to $d_{0}$.

$$
\Delta F\left(\omega_{0} t\right) \cong \frac{t_{d a m p}}{T_{S}} \frac{2 \varepsilon_{0} \varepsilon_{r} A}{d_{0}^{2}} V_{B} V_{c t r l} \sin \left(\omega_{0} t\right)
$$

Therefore, if the design aim is to have the fastest transducer settling time, a maximum amount of electrostatic force needs to be applied to the proof mass. From (6) it can be seen that there are several parameters which can be adjusted in order to increase the electrostatic force: the excitation electrodes surface, the control voltage and the damping duty cycle. Assuming that the MEMS design parameters are fixed and the maximum control voltage that can be applied is fixed by a certain technology power supply, the only parameter which can vary is the damping duty cycle or the electrostatic force application duration.

The novel architecture shown in Fig.2 proposes a method to improve the damping duty cycle for MEMS that can sense up to three degrees of freedom.

The transducer can be modeled by a second order mass spring transfer function $H_{\text {sens }}(s)$ where $\omega_{0}$ is the MEMS natural pulsation. Moreover, the external acceleration applied to the sensor can be read using the capacitance variation $\Delta C$ that appears when the proof mass moves. During the measurement phase $\Phi 1$, due to the voltage applied on the MEMS electrodes, a charge variation $\Delta Q$ is injected into a Charge to Voltage converter $(\mathrm{C} 2 \mathrm{~V})$ which provides the voltage $V$ to the control block. The particularity of this architecture is that the $\mathrm{C} 2 \mathrm{~V}$ is shared between the 3-axis, which allows a low power and small area architecture. However, since the $\mathrm{C} 2 \mathrm{~V}$ as well as the sensor middle plate are shared, only a single axis measurement can be performed at a time. For example, if during the $x$-axis measurement phase, a voltage is applied on the $y$-axis electrodes, then parasitical charges will be injected into the $\mathrm{C} 2 \mathrm{~V}$ and the $x$-axis acceleration measurement will be corrupted.

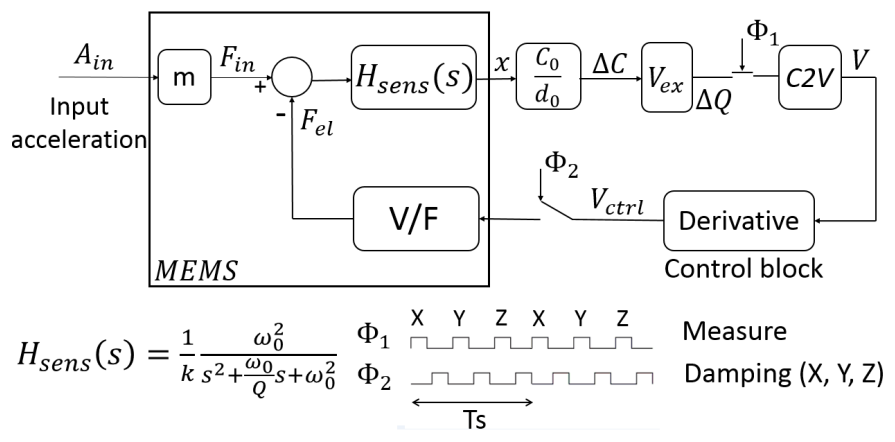

Fig.2 System block diagram

After the $\mathrm{C} 2 \mathrm{~V}$, a control block implementing the derivative, is required, to provide an estimation of the velocity. As the $\mathrm{C} 2 \mathrm{~V}$ output reflects the proof mass displacement, the velocity estimation can thus be obtained by computing the difference between two successive $\mathrm{C} 2 \mathrm{~V}$ output samples (7).

$$
V_{c t r l}\left(n T_{s}\right)=k d *\left(V\left(n T_{s}\right)-V\left((n-1) T_{s}\right)\right.
$$

where $\mathrm{kd}$ is a derivative gain and a design parameter.

During the damping phase, $\Phi 2$, the control voltage which was previously calculated, is applied on MEMS excitation electrodes. The control voltage can be sampled and hold by the 
derivative block, thanks to designed memory capacity, during the entire sampling period $T_{S}$.

Furthermore, each axis has its own control block and three derivative signals, corresponding to each of the three axis, can be applied simultaneously on the MEMS electrodes when the damping phase occurs.

\section{SIMULTANEOUS DAMPING CONTROL}

The 3-axis underdamped accelerometer MEMS, with the read-out interface and the control blocks is shown in Fig.3. The MEMS charge variation can be measured using an inverting amplifier and a feedback capacitor $C_{f b}$ which will be systematically reset between each axis measurement. The voltage applied on the MEMS excitation electrodes, will depend on the system operating phase (measurement or damping). Due to the $\mathrm{C} 2 \mathrm{~V}$ feedback, $V_{m}$ is applied on the MEMS middle plate during all phases. Moreover, the time multiplexed electrodes structure can be translated into a discrete system with simultaneous and multi-rate damping control. We define $T_{S}$ the system sampling period or the time during which all 3-axis have been measured and damped. Each of the three derivative blocks will update their output value, and therefore the damping force, only once per period $T_{S}$.

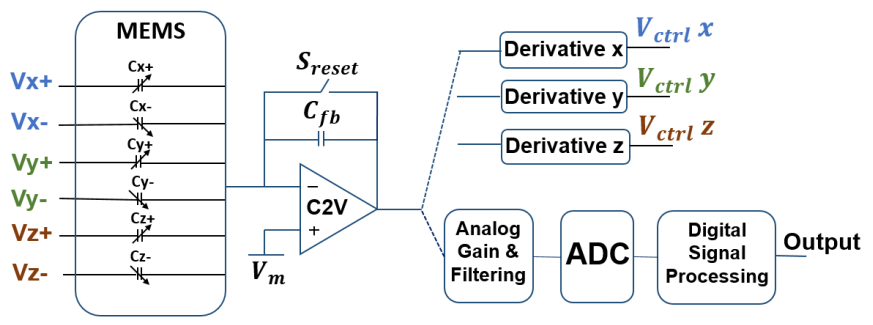

Fig.3 Three axis closed loop underdamped accelerometer

A novel sequence, which optimizes the damping efficiency, has been designed and implemented (Fig.4). Six separate phases can be distinguished in the same sampling period Ts. For the 3-axis: $x, y$ and $z$, the system has three reading and three damping phases. During Phase 1, on the $x$ positive excitation electrode a $2 \mathrm{~V}_{\mathrm{m}}$ voltage is applied while the $x$ negative excitation electrode is connected to the analog ground. In this way, the charge variation, caused by the proof mass movement, is transferred into the $\mathrm{C} 2 \mathrm{~V}$. Additionally, after reading $x$-axis acceleration, the Derivative $x$ control block performs the difference between two successive $\mathrm{C} 2 \mathrm{~V}$ output $x$ samples and adds $\mathrm{a} \pm \mathrm{V}_{\mathrm{B}}$ voltage to the output signal. When Phase 2 starts, the new $x$-axis velocity estimation, which has just been calculated during Phase 1 , is applied on $x$-axis MEMS excitation electrodes introducing an artificial coefficient $\mathrm{B}$ in (2) and increasing the $x$ damping coefficient. During the same phase, the $y$ and $z$ velocity estimations, that have been previously calculated and stored during the $(n-1)$ Ts sampling period, can be applied on $y$ and $z$ excitation electrodes.

When Phase 3 occurs, $2 \mathrm{~V}_{\mathrm{m}}$ and $0 \mathrm{~V}$ are applied on $y$-axis excitation electrodes and a new $\mathrm{C} 2 \mathrm{~V}$ output $y$ sample will be available. A new damping force value is calculated and applied during Phase 4. Furthermore, the same $x$ damping force which was firstly applied during Phase 2 but still stored using the $x$ control block, and the $z$ damping force which was calculated in the previous sampling period $(n-1) T_{s}$, are applied on MEMS electrodes.

\begin{tabular}{|c|c|c|c|c|c|c|c|c|}
\hline & $\begin{array}{c}\text { Phase } \\
1 \\
\end{array}$ & $\begin{array}{c}\text { Phase } \\
2 \\
\end{array}$ & $\begin{array}{c}\text { Phase } \\
3 \\
\end{array}$ & $\begin{array}{c}\text { Phase } \\
4\end{array}$ & $\begin{array}{c}\text { Phase } \\
5 \\
\end{array}$ & $\begin{array}{c}\text { Phase } \\
6\end{array}$ & $\begin{array}{c}\text { Phase } \\
1 \\
\end{array}$ & $\begin{array}{c}\text { Phase } \\
2 \\
\end{array}$ \\
\hline$X$ & $\begin{array}{c}\text { Read } \\
x\end{array}$ & $\begin{array}{c}\text { Damp } \\
x[n]\end{array}$ & 0 & $\begin{array}{c}\text { Damp } \\
x[n]\end{array}$ & 0 & $\begin{array}{c}\text { Damp } \\
x[n]\end{array}$ & $\begin{array}{c}\text { Read } \\
x\end{array}$ & $\begin{array}{l}\text { Damp } \\
x[n+1]\end{array}$ \\
\hline $\mathbf{Y}$ & 0 & $\begin{array}{l}\text { Damp } \\
y[n-1]\end{array}$ & $\begin{array}{c}\text { Read } \\
y\end{array}$ & $\begin{array}{c}\text { Damp } \\
\mathrm{y}[\mathrm{n}]\end{array}$ & 0 & $\begin{array}{c}\text { Damp } \\
y[n]\end{array}$ & 0 & $\begin{array}{c}\text { Damp } \\
\mathrm{y}[\mathrm{n}]\end{array}$ \\
\hline $\mathbf{Z}$ & 0 & $\begin{array}{l}\text { Damp } \\
\text { z[n-1] }\end{array}$ & 0 & $\begin{array}{l}\text { Damp } \\
\text { z[n-1] }\end{array}$ & $\begin{array}{c}\text { Read } \\
z\end{array}$ & $\begin{array}{c}\text { Damp } \\
z[n]\end{array}$ & 0 & $\begin{array}{c}\text { Damp } \\
\text { z[n] }\end{array}$ \\
\hline
\end{tabular}

Fig.4 Novel sequence description

Finally, during Phase 5, a new $z$-axis acceleration value is measured and a new $z$ axis control signal will be available. This new value will then be applied on $z$-axis excitation electrodes when Phase 6 occurs. During the same Phase 6, the same $x$ damping force which was firstly applied during Phase 2 and the $y$ damping force which was firstly applied during Phase 4, are also applied on MEMS electrodes.

In this way, when one sampling period is complete, the three axes were measured and damped and three times more electrostatic force was applied to the transducer. When the amount of electrostatic force applied is increased so is the damping coefficient and the sensor ability to oscillate is diminished. As soon as the $\mathrm{C} 2 \mathrm{~V}$ output reaches the steady state, its output voltage can be sent to the Analog to Digital Converter (ADC) and the signal processing chain in order to be processed as a valid acceleration measurement data. Consequently, the main parameter one would like to improve is the system settling time or the time that the $\mathrm{C} 2 \mathrm{~V}$ needs, before sending a valid data to the output.

\section{SIMULATIONS RESULTS}

The architecture presented in Fig. 3 was fully modeled and simulated using Matlab - Simulink. The accelerometer MEMS movement was modeled with the functioning equation (1) and the capacitance variation $\Delta C$ is calculated using the proof mass displacement $x(8)$.

$$
\Delta C=\frac{\varepsilon_{0} \varepsilon_{r} A}{d_{0}} x
$$

Sample and hold techniques were employed to model system sequences and depending on the phase, a different voltage level is applied on the electrodes. The MEMS natural frequency is $f_{0}=4 \mathrm{kHz}$ and the open loop quality factor Q is 2000. An external acceleration of $1 \mathrm{~g}=9.8 \mathrm{~m} / \mathrm{s}^{2}$ is applied and results in a proof mass displacement of $x=15.9 \mathrm{~nm}$.

For a feedback capacitor of $C_{f b}=300 \mathrm{fF}$, the $\mathrm{C} 2 \mathrm{~V}$ is able to output $V=13 \mathrm{mV}$ during the steady state regime under a $1.75 \mathrm{~V}$ power supply. However, if no additional damping is 
applied and the MEMS quality factor is 2000 , the system settling time is $50 \mathrm{~ms}$.

Firstly, the new damping algorithm was tested. It can be seen in Fig. 5 that under the effect of $1 g$ extern acceleration in the $x$ direction, for example, the proof mass will slightly oscillate due to the electrostatic damping control. Moreover, from one single control voltage sample $V_{c t r l} x$, which is generated at each sampling period, the same electrostatic force is applied during the three damping phases. Note that the electrostatic force value will be null except during the damping phases.

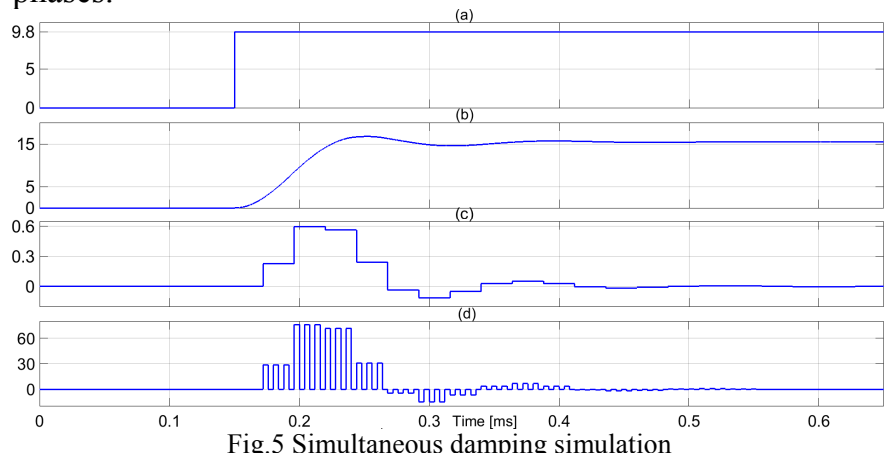

(a) Input acceleration Ain=1g (b) MEMS displacement [nm]

(c) Control voltage $\left(V_{c t r l} x\right)$ [V] (d) Electrostatic force [nN]

Secondly, to assess the efficiency of the proposed simultaneous damping method, a chronogram of a more classical approach (successive damping) was implemented (Fig.6) for the same architecture from Fig.3. The results were compared to those obtained using the chronograms from Fig.4.

\begin{tabular}{|c|c|c|c|c|c|c|c|c|}
\hline & $\begin{array}{c}\text { Phase } \\
1\end{array}$ & $\begin{array}{c}\text { Phase } \\
2\end{array}$ & $\begin{array}{c}\text { Phase } \\
3\end{array}$ & $\begin{array}{c}\text { Phase } \\
4\end{array}$ & $\begin{array}{c}\text { Phase } \\
5\end{array}$ & $\begin{array}{c}\text { Phase } \\
6\end{array}$ & $\begin{array}{c}\text { Phase } \\
1\end{array}$ & $\begin{array}{c}\text { Phase } \\
2\end{array}$ \\
\hline$X$ & $\begin{array}{c}\text { Read } \\
x\end{array}$ & $\begin{array}{c}\text { Damp } \\
x[n]\end{array}$ & 0 & 0 & 0 & 0 & $\begin{array}{c}\text { Read } \\
X\end{array}$ & $\begin{array}{l}\text { Damp } \\
x[n+1]\end{array}$ \\
\hline$Y$ & 0 & 0 & $\begin{array}{c}\text { Read } \\
y\end{array}$ & $\begin{array}{c}\text { Damp } \\
\text { y[n] }\end{array}$ & 0 & 0 & 0 & 0 \\
\hline Z & 0 & 0 & 0 & 0 & $\begin{array}{c}\text { Read } \\
z\end{array}$ & $\begin{array}{c}\text { Damp } \\
\text { z[n] }\end{array}$ & 0 & 0 \\
\hline
\end{tabular}

Fig.6 Successive damping sequence

Considering now the chronograms from Fig.6, it can be seen that the measurement and the damping phases for all three axis are successively occurring. Each sampling period has only one damping phase for each axis, thereby the electrostatic force duty cycle is reduced by three compared to the method proposed in this paper.

The system settling time depends both on the amount of electrostatic force applied and on the sampling frequency. In order to compare the two damping chronograms performances, the sampling period was varied between 6 us and $36 u s$ and the derivative gain $k d$ fixed. Fig. 7 presents the settling time results measured in both situations: simultaneous and successive damping.

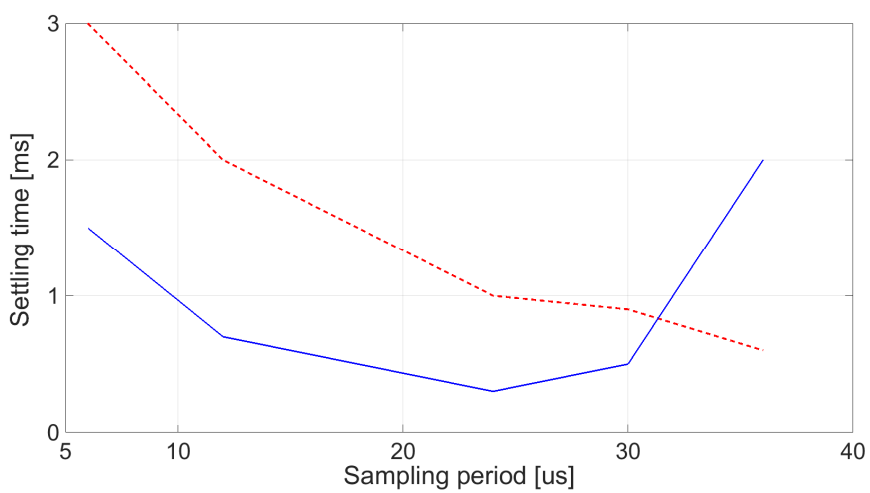

Fig.7 Settling time for simultaneous damping (solid blue line) and successive damping (dotted red line)

From Fig.7, it can be seen that at high sampling frequencies $\left(T_{s}=6 u s\right)$ the simultaneous damping is very efficient and the settling time is roughly three times smaller than for the successive damping. When the system sampling frequency becomes slower $\left(T_{s}=30 u s\right)$ the successive damping may be a pertinent solution if the design aim is the response settling time.

\section{CONCLUSION}

A novel simultaneous damping architecture for a 3-axis high Q MEMS accelerometer was proposed. Damping coefficient is increased and system settling time can be improved by simply storing and applying the same amount of electrostatic force during several phases in the same sampling period. Two different approaches are considered for physical validation: ASIC design or discrete control loop.

The application of this novel design can be the multiple degrees of freedom systems requiring very fast responses.

\section{ACKNOWLEDGMENT}

The authors would like to thank NXP Semiconductors for its help and contribution.

\section{REFERENCES}

[1] M. Kraft, C.P. Lewis, T.G. Hesketh, "Closed-loop silicon accelerometers" Circuits, Devices and Systems, IEEE Proceedings, vol. 145 , issue 5, Oct 1998 .

[2] Fang Chen, Weizheng Yuan, Honglong Chang, I. Zeimpekis, M.Kraft "Low noise vacuum MEMS closed-loop accelerometer using sixth-order multi-feedback loops and local resonator sigma delta modulator" 2014 IEEE $27^{\text {th }}$ International conference on Micro Electro Mechanical systems (MEMS) , Jan. 2014

[3] Honglin $\mathrm{Xu}$, Xiaowei Liu, Liang Yin "A Closed-loop $\Sigma \Delta$ Interface for a High Q Micromechanical Capacitive Accelerometer With $200 \mathrm{ng} / \sqrt{\mathrm{Hz}}$ Input Noise Density" IEEE Journal of Solid-State Circuits, vol. 50, issue 9, Sept.2015.

[4] M. Yucetas, M. Pulkkinen, A. Kalanti, J. Salomaa "A High-Resolution Accelerometer With Electrostatic Damping and Improved Supply Sensitivity" IEEE Journal of Solid-State Circuits, vol. 47, issue 7, July 2012.

[5] Z. Ye, H. Yang, T. Yin, G. Huang "High-Performance Closed-Loop Interface Circuit for High-Q Capacitive Microaccelerometers" IEEE Sensors Journal, vol.13, issue 5, May 2013. 